\title{
THE EFFECTS OF DIFFERENT TYPES OF STIMULUS AND ITS TEST-RETEST RELIABILITY ON CERVICAL EVOKED MYOGENIC POTENTIAL (CVEMP) RESULTS
}

\author{
Mohamad Amir Faisal Mohd Saufi ${ }^{1}$, Nur Hafizah Sulaiman ${ }^{1}$ and Sarah Rahmat ${ }^{1}$ \\ ${ }^{1}$ Department of Audiology and Speech-language Pathology, Kuliyyah of Allied Health \\ Science, International Islamic University of Malaysia, Kuantan, Pahang.
}

Presenter: Mohamad Amir Faisal Mohd Saufi, amirfaisalsaufi@gmail.com

Introduction: The Cervical Vestibular Evoked Myogenic Potential (cVEMP) is used to evaluate the integrity of saccule and inferior vestibular nerve. There are a lot of factors affecting CVEMP results including stimulus types. This study was carried out to determine the effects of different stimuli on CVEMP results and its test-retest reliability.

Materials and method: 25 normal hearing subjects were recruited. The cVEMP testing were performed in 2 sessions with 1 week gap between each sessions. The CVEMP waveforms were recorded in sitting upright position with electrodes placed at; i) upper one-third of sternocleidomastoid muscle for active electrode, ii) suprasternal notch for inactive electrode and, iii) middle of forehead for the ground electrode. The stimuli ( $500 \mathrm{~Hz}$ tone burst, click, narrowband chirp and broadband chirp) were presented via insert phone at 95dBnHL. The cVEMP results ( $\mathrm{P} 13-\mathrm{N} 23$ peak-to-peak amplitude, P13 latency and N23 latency) were recorded.

Results: Result showed; i) $500 \mathrm{~Hz}$ tone burst produced significantly largest amplitude; ii) narrowband chirp produced significantly shortest P13 latency; iii) broadband chirp produced significantly shortest N23 latency; iv) no significant difference of P13 and N23 latency were observed between two sessions; and v) significant difference of P13-N23 amplitude were observed between two sessions for all stimuli except for narrowband chirp.

Conclusion: The $500 \mathrm{~Hz}$ tone burst was observed to be the most ideal stimulus (produce highest amplitude). All stimuli produced good test-retest reliability in terms of latency. However, most of the stimuli produced poor test-retest reliability in terms of amplitude except for narrowband chirps. 